\title{
Acceleration of Preconditioned Krylov Solvers for Bubbly Flow Problems
}

\author{
J.M. Tang and C. Vuik \\ Delft University of Technology, \\ Faculty of Electrical Engineering, Mathematics and Computer Science, \\ Delft Institute of Applied Mathematics, \\ Mekelweg 4, 2628 CD Delft, The Netherlands \\ $\{$ j.m.tang, c.vuik\}@tudelft.nl
}

\begin{abstract}
We consider the linear system which arises from discretization of the pressure Poisson equation with Neumann boundary conditions, coming from bubbly flow problems. In literature, preconditioned Krylov iterative solvers are proposed, but these show slow convergence for relatively large and complex problems. We extend these traditional solvers with the so-called deflation technique, which accelerates the convergence substantially. Several numerical aspects are considered, like the singularity of the coefficient matrix and the varying density field at each time step. We demonstrate theoretically that the resulting deflation method accelerates the convergence of the iterative process. Thereafter, this is also demonstrated numerically for 3-D bubbly flow applications, both with respect to the number of iterations and the computational time.
\end{abstract}

Keywords: deflation, conjugate gradient method, preconditioning, symmetric positive semi-definite matrices, bubbly flow problems.

\section{Introduction}

Recently, moving boundary problems have received much attention in literature, due to their applicative relevance in many physical processes. One of the most popular moving boundary problems is modelling bubbly flows, see e.g. 12 . These bubbly flows can be simulated, by solving the well-known Navier-Stokes equations for incompressible flow:

$$
\left\{\begin{array}{l}
\frac{\partial u}{\partial t}+u \cdot \nabla u+\frac{1}{\rho} \nabla p=\frac{1}{\rho} \nabla \cdot \mu\left(\nabla u+\nabla u^{T}\right)+g \\
\nabla \cdot u=0
\end{array}\right.
$$

where $g$ represents the gravity and surface tension force, and $\rho, p, \mu$ are the density, pressure and viscosity, respectively. Eqs. (11) can be solved using, for instance, the pressure correction method [7]. The most time-consuming part of this method is solving the symmetric and positive semi-definite (SPSD) linear system on each time step, which comes from a second-order finite-difference 
discretization of the Poisson equation with possibly discontinuous coefficients and Neumann boundary conditions:

$$
\left\{\begin{aligned}
\nabla \cdot\left(\frac{1}{\rho} \nabla p\right) & =f_{1}, & & \mathbf{x} \in \Omega, \\
\frac{\partial}{\partial \mathbf{n}} p & =f_{2}, & & \mathbf{x} \in \partial \Omega,
\end{aligned}\right.
$$

where $\mathbf{x}$ and $\mathbf{n}$ denote the spatial coordinates and the unit normal vector to the boundary $\partial \Omega$, respectively. In the $3-\mathrm{D}$ case, domain $\Omega$ is chosen to be a unit cube. Furthermore, we consider two-phase bubbly flows, so that $\rho$ is piecewise constant with a relatively high contrast:

$$
\rho= \begin{cases}\rho_{0}=1, & \mathbf{x} \in \Lambda_{0}, \\ \rho_{1}=10^{-3}, & \mathbf{x} \in \Lambda_{1},\end{cases}
$$

where $\Lambda_{0}$ is water, the main fluid of the flow around the air bubbles, and $\Lambda_{1}$ is the region inside the bubbles.

The resulting linear system which has to be solved is

$$
A x=b, \quad A \in \mathbb{R}^{n \times n},
$$

where the singular coefficient matrix $A$ is $\operatorname{SPSD}$ and $b \in \operatorname{range}(A)$. In practice, the preconditioned Conjugate Gradient (CG) method [4 is widely used to solve (4), see also References [1, 2, 3, 5]. In this paper, we will restrict ourselves to the Incomplete Cholesky (IC) decomposition [8] as preconditioner, and the resulting method will be denoted as ICCG. In this method,

$$
M^{-1} A x=M^{-1} b, \quad M \text { is the IC preconditioner, }
$$

is solved using CG. ICCG shows good performance for relatively small and easy problems. For complex bubbly flows or for problems with large jumps in the density, this method shows slow convergence, due to the presence of small eigenvalues in the spectrum of $M^{-1} A$, see also 13 .

To remedy the bad convergence of ICCG, the deflation technique has been proposed, originally from Nicolaides 11. The idea of deflation is to project the extremely small eigenvalues of $M^{-1} A$ to zero. This leads to a faster convergence of the iterative process, due to the fact that CG can handle matrices with zeroeigenvalues [6] and the effective condition number becomes more favorable. The resulting method is called Deflated ICCG or shortly DICCG, following [17, and it will be further explained in the next section.

\section{DICCG Method}

In DICCG, we solve

$$
M^{-1} P A \tilde{x}=M^{-1} P b, \quad P \text { is the deflation matrix, }
$$

using CG, where

$$
P:=I-A Z E^{-1} Z^{T}, \quad E:=Z^{T} A Z, \quad Z \in \mathbb{R}^{n \times r}, \quad r \ll n .
$$


Piecewise-constant deflation vectors are used to approximate the eigenmodes corresponding to the components which caused the slow convergence of ICCG. More technically, deflation subspace matrix $Z=\left[\begin{array}{llll}z_{1} & z_{2} & \cdots & z_{r}\end{array}\right]$ consists of deflation vectors $z_{j}$ with

$$
z_{j}(\mathbf{x})= \begin{cases}0, & \mathbf{x} \in \Omega \backslash \bar{\Omega}_{j} \\ 1, & \mathbf{x} \in \Omega_{j},\end{cases}
$$

where the domain $\Omega$ is divided into non-overlapping subdomains $\Omega_{j}$, which are chosen to be cubes, assuming that the number of grid points in each spatial direction is the same. Note that, due to the construction of the sparse matrix $Z$, matrices $A Z$ and $E$ are sparse as well, so that the extra computations with the deflation matrix $P$ are relatively cheap.

\section{Application of DICCG to Bubbly Flow Problems}

The deflation technique works well for invertible systems and when the deflation vectors are based on the geometry of the problem, see also References [9, 10, Main questions in this paper are:

- is the deflation method also applicable to linear systems with singular matrices?

- is the deflation method with fixed deflation vectors also applicable to problems, where the position and radius of the bubbles change in every time step?

The second question will be dealt in the next section, where numerical experiments will be presented to show the success of the method for time-dependent bubbly flow problems.

First, we show that DICCG can be used for singular matrices. Due to the construction of matrix $Z$ and the singularity of $A$, the coarse matrix $E:=Z^{T} A Z$ is also singular. In this case, $E^{-1}$ does not exist. We propose several new variants of deflation matrices $P$ :

( $i$ ) invertibility of $A$ is forced resulting in a deflation matrix $P_{1}$, i.e., we adapt the last element of $A$ such that the new matrix, denoted as $\widetilde{A}$, is invertible;

(ii) a column of $Z$ is deleted resulting in a deflation matrix $P_{2}$, i.e., instead of $Z$ we take $\left[\begin{array}{llll}z_{1} & z_{2} & \cdots & z_{r-1}\end{array}\right]$ as the deflation subspace matrix;

(iii) systems with a singular $E$ are solved iteratively resulting in a deflation matrix $P_{3}$, i.e., matrix $E^{-1}$, as given in Eq. (5), is considered to be a pseudo-inverse.

As a result, Variant $(i)$ and $(i i)$ give a non-singular matrix $E$ and, in addition, the real inverse of $E$ is not required anymore in Variant (iii). Subsequently, we can prove that the three DICCG variants are identical in exact arithmetic, see Theorem [1]

Theorem 1. $P_{1} \widetilde{A}=P_{2} A=P_{3} A$.

Proof. The proof can be found in [15, 16. 
We observe that the deflated systems of all variants are identical. From this result, it is easy to show that the preconditioned deflated systems are also the same. Since the variants are equal, any of them can be chosen in the numerical experiments. We will apply the first variant for convenience, and the results and efficiency of this variant will be demonstrated numerically, in the next section.

\section{Numerical Experiments}

We test the efficiency of the DICCG method for two kind of test problems.

\subsection{Test Case 1: Stationary Problem}

First, we take a 3-D bubbly flow application with eight air-bubbles in a domain of water, see Figure 1 for the geometry. We apply finite differences on a uniform Cartesian grid with $n=100^{3}$, resulting in a very large but sparse linear system $A x=b$ with SPSD matrix $A$.

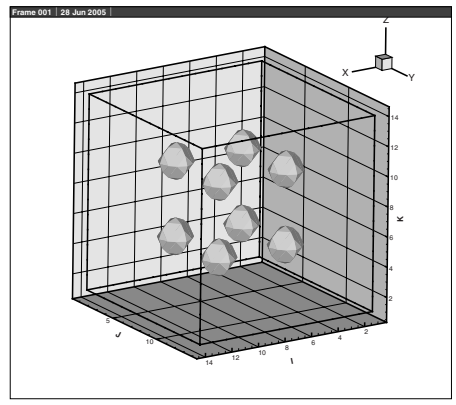

Fig. 1. An example of a bubbly flow problem: eight air-bubbles in a unit domain filled with water

Then, the results of ICCG and DICCG can be found in Table 1, where $\phi$ denotes the final relative exact residual and DICCG $-r$ denotes DICCG with $r$ deflation vectors. Moreover, we terminate the iterative process, when the relative update residuals are smaller than the stopping tolerance $\epsilon=10^{-8}$.

From Table 1, one observes that the larger the number of deflation vectors, the less iterations DICCG requires. With respect to the CPU time, there is an optimum, namely for $r=10^{3}$. Hence, in the optimal case, DICCG is more than five times faster compared to the original ICCG method, while the accuracy of both methods are comparable!

Similar results also hold for other related test cases. Results of ICCG and DICCG for the problem with 27 bubbles can be found in Table2 In addition, it appears that the benefit of the deflation method is larger when we increase the number of grid points, $n$, in the test cases, see also [16. 
Table 1. Convergence results of ICCG and DICCG $-r$ solving $A x=b$ with $n=100^{3}$, for the test problem as given in Figure 1

\begin{tabular}{l|ll|l}
\hline \hline Method & \# Iterations & CPU Time (s) & $\phi\left(\times 10^{-9}\right)$ \\
\hline \hline ICCG & 291 & 43.0 & 1.1 \\
DICCG $-2^{3}$ & 160 & 29.1 & 1.1 \\
DICCG $-5^{3}$ & 72 & 14.2 & 1.2 \\
DICCG $-10^{3}$ & $\mathbf{3 6}$ & $\mathbf{8 . 2}$ & $\mathbf{0 . 7}$ \\
DICCG $-20^{3}$ & 22 & 27.2 & 0.9 \\
\hline \hline
\end{tabular}

Table 2. Convergence results of ICCG and DICCG $-r$ solving $A x=b$ with $n=100^{3}$, for the test case with 27 bubbles

\begin{tabular}{l|ll|l}
\hline \hline Method & \# Iterations & CPU Time (sec) & $\phi\left(\times 10^{-9}\right)$ \\
\hline \hline ICCG & 310 & 46.0 & 1.3 \\
DICCG $-2^{3}$ & 275 & 50.4 & 1.3 \\
DICCG $-5^{3}$ & 97 & 19.0 & 1.2 \\
DICCG $-10^{3}$ & $\mathbf{6 0}$ & $\mathbf{1 3 . 0}$ & $\mathbf{1 . 2}$ \\
DICCG $-20^{3}$ & 31 & 29.3 & 1.2 \\
\hline \hline
\end{tabular}

Finally, for the test case with 27 bubbles, the plots of the residuals during the iterative process of both ICCG and DICCG can be found in Figure 2. Notice that the behavior of the residuals of ICCG are somewhat irregularm due to the presence of the bubbles. For DICCG, we conclude that the larger $r$, the more linear the residual plot is, so the faster the convergence of the iterative process. Apparently, the eigenvectors associated to the small eigenvalues of $M^{-1} A$ have been well-approximated by the deflation vectors, if $r$ is sufficiently large.

\subsection{Test Case 2: Time-Dependent Problem}

Next, we present some results from the 3-D simulation of a rising air bubble in water, in order to show that the deflation method is also applicable to reallife problems with varying density fields. We adopt the mass-conserving levelset method 13 for the simulations, but it could be replaced by any operatorsplitting method, in general. At each time step, a pressure Poisson equation has to be solved, which is the most time-consuming part of the whole simulation. Therefore, during this section we only concentrate on this part at each time step. We investigate whether DICCG is efficient for all those time steps.

We consider a test problem with a rising air bubble in water without surface tension. The exact material constants and other relevant information can be found in [13, Sect. 8.3.2]. The starting position of the bubble in the domain and the evolution of the movement during the 250 time steps are given in Figure 3 .

In [13, the Poisson solver is based on ICCG. Here, we will compare this method to DICCG with $r=10^{3}$ deflation vectors, in the case of $n=100^{3}$. The results are presented in Figure 4. 


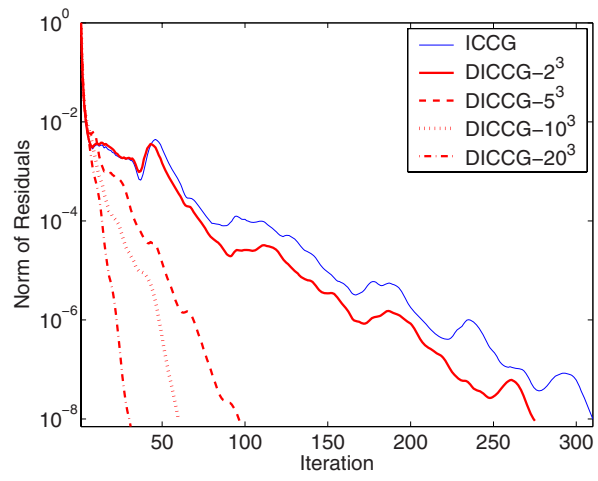

Fig. 2. Residual plots of ICCG and DICCG $-r$, for the test problem with 27 bubbles and various number of deflation vectors $r$

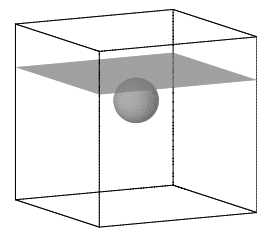

(a) $t=0$.

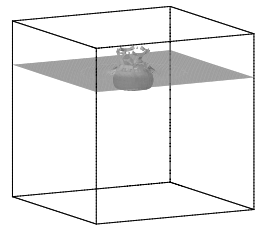

(d) $t=150$.

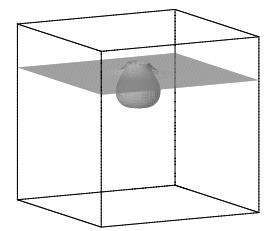

(b) $t=50$.

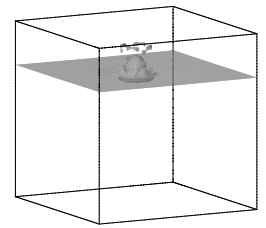

(e) $t=200$.

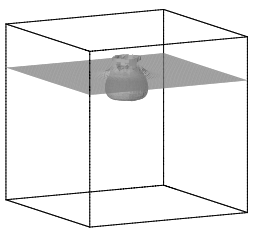

(c) $t=100$.

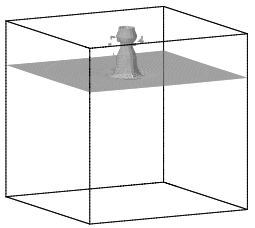

(f) $t=250$.

Fig. 3. Evolution of the rising bubble in water without surface tension in the first 250 time steps

From Subfigure 4(a), we notice that the number of iterations is strongly reduced by the deflation method. DICCG requires approximately 60 iterations, while ICCG converges between 200 and 300 iterations at most time steps. Moreover, we observe the erratic behavior of ICCG, whereas DICCG seems to be less sensitive to the geometries during the evolution of the simulation. Also with respect of the CPU time, DICCG shows very good performance, see Subfigure 4(b) At most time steps, ICCG requires 25-45 seconds to converge, whereas DICCG only needs around 11-14 seconds. Moreover, in Figure 4(c) one can find the gain factors, considering both the ratios of the iterations and the CPU time between ICCG and DICCG. From this figure, it can be seen that DICCG needs approximately 4-8 times less iterations, depending on the time step. More important, 


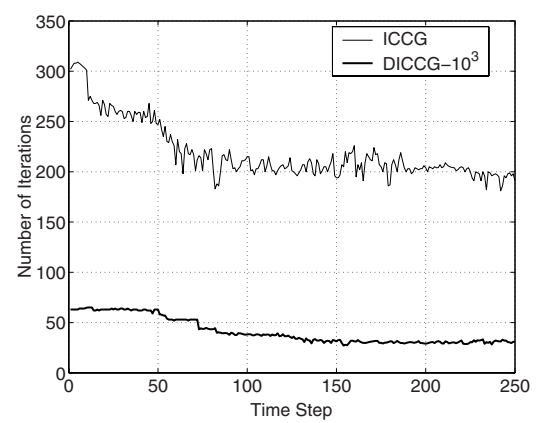

(a) Number of iterations versus time step.

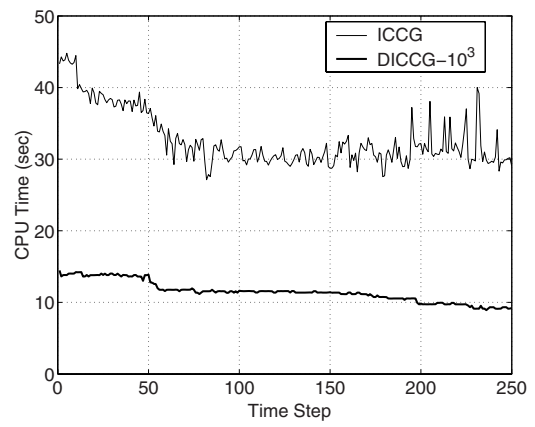

(b) CPU time versus time step.

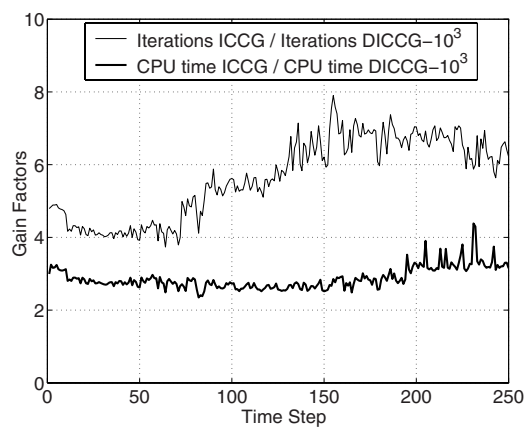

(c) Gain factors with respect to ICCG and DICCG.

Fig. 4. Results of ICCG and DICCG with $r=10^{3}$, for the simulation with a rising air bubble in water

DICCG converges approximately 2-4 times faster to the solution compared to ICCG, at all time steps.

In general, we see that, compared to ICCG, DICCG decreases significantly the number of iterations and the computational time as well, which are required for solving the pressure Poisson equation with discontinuous coefficients, in applications of 3-D bubbly flows.

\section{Conclusions}

A deflation technique has been proposed to accelerate the convergence of standard preconditioned Krylov methods, for solving bubbly flow problems. In literature, this deflation method has already been proven to be efficient,for linear systems with invertible coefficient matrix and not-varying density fields in time. However, in our bubbly flow applications, we deal with linear systems with a singular matrix and varying density fields. In this paper, we have shown, both 
theoretically and numerically, that the deflation method with fixed subdomain deflation vectors can also be applied to this kind of problems. The method appeared to be robust and very efficient in various numerical experiments, with respect to both the number of iterations and the computational time.

\section{References}

1. Benzi, M.: Preconditioning techniques for large linear systems: A survey, J. Comp. Phys., 182 (2002), 418-477

2. Gravvanis, G.A.: Explicit Approximate Inverse Preconditioning Techniques, Arch. Comput. Meth. Eng., 9 (2002), 371-402.

3. Grote, M.J., Huckle, T.: Parallel preconditioning with sparse approximate inverses, SIAM J. Sci. Comput., 18 (1997), 838-853.

4. Hestenes, M.R., Stiefel, E.: Methods of Conjugate Gradients for Solving Linear Systems, J. Res. Nat. Bur. Stand., 49 (1952), 409-436.

5. Huckle, T.: Approximate sparsity patterns for the inverse of a matrix and preconditioning, Appl. Num. Math., 30 (1999), 291-303.

6. Kaasschieter, E.F.: Preconditioned Conjugate Gradients for solving singular systems, J. Comp. Appl. Math., 24 (1988), 265-275.

7. Kan, J.J.I.M. van: A second-order accurate pressure correction method for viscous incompressible flow, SIAM J. Sci. Stat. Comp., 7 (1986), 870-891.

8. Meijerink, J.A., Vorst, H.A. van der: An iterative solution method for linear systems of which the coefficient matrix is a symmetric M-matrix, Math. Comp., 31 (1977), 148-162.

9. Nabben, R., Vuik, C.: A comparison of Deflation and Coarse Grid Correction applied to porous media flow, SIAM J. Numer. Anal., 42 (2004), 1631-1647.

10. Nabben, R., Vuik, C.: A Comparison of Deflation and the Balancing Preconditioner, SIAM J. Sci. Comput., 27 (2006), 1742-1759.

11. Nicolaides, R.A.: Deflation of Conjugate Gradients with applications to boundary value problems, SIAM J. Matrix Anal. Appl., 24 (1987), 355-365.

12. Van der Pijl, S.P., Segal, A., Vuik, C., Wesseling, P.: A mass-conserving Level-Set method for modelling of multi-phase flows, Int. J. Num. Meth. in Fluids, 47(4) (2005), 339-361.

13. Van der Pijl, S.P.: Computation of bubbly flows with a mass-conserving level-set method, PhD thesis, Delft University of Technology, Delft (2005).

14. Sousa, F.S., Mangiavacchi, N., Nonato, L.G., Castelo, A., Tome, M.F., Ferreira, V.G., Cuminato, J.A., McKee, S.: A Front-Tracking / Front-Capturing Method for the Simulation of 3D Multi-Fluid Flows with Free Surfaces, J. Comp. Physics, 198 (2004), 469-499.

15. Tang, J.M., Vuik, C.: On Deflation and Singular Symmetric Positive Semi-Definite Matrices, J. Comp. Appl. Math., to appear (2006).

16. Tang, J.M., Vuik, C.: An Efficient Deflation Method applied on 2-D and 3-D Bubbly Flow Problems, Elec. Trans. Num. Anal., submitted (2006).

17. Vuik, C., Segal, A., Meijerink, J.A.: An efficient preconditioned CG method for the solution of a class of layered problems with extreme contrasts in the coefficients, J. Comp. Phys., 152 (1999), 385-403. 